\title{
An Energy Detection-Based Spectrum-Sensing Method for Cognitive Radio
}

\author{
Jun Luo iD, ${ }^{1,2}$ Guoping Zhang, ${ }^{2}$ and Chiyu Yan ${ }^{3}$ \\ ${ }^{1}$ Xinyu University, Xiny 338004, China \\ ${ }^{2}$ Key Laboratory of Quark and Lepton Physics (MOE) and College of Physics Science and Technology, \\ Central China Normal University, Wuhan 430079, China \\ ${ }^{3}$ School of Information Science and Technology, Tsinghua University, Beijing 100084, China \\ Correspondence should be addressed to Jun Luo; 342086803@qq.com
}

Received 8 November 2021; Revised 30 January 2022; Accepted 4 February 2022; Published 23 February 2022

Academic Editor: Francisco R. Castillo-Soria

Copyright (c) 2022 Jun Luo et al. This is an open access article distributed under the Creative Commons Attribution License, which permits unrestricted use, distribution, and reproduction in any medium, provided the original work is properly cited.

\begin{abstract}
Energy detection (ED) method is one of the most commonly used signal-sensing methods in spectrum sensing due to its low implementation complexity. ED can achieve good detection performance when the noise variance is known. However, in most cases, the noise variance is estimated, which may result in the uncertainty in noise variance. In the presence of noise variance uncertainty, the detection performance of the ED method may degrade significantly. To reduce the impact of uncertainty in noise variance, an ED-based sensing method is proposed in this paper. This method combines these signal samples sampled by multiple antennas to obtain the decision statistic as ED does. A novel technique is proposed to construct the decision threshold so that it is independent of the noise variance. As a consequence, the proposed method is free of the effect of noise variance uncertainty, and the noise variance estimation is not need. The simulation results show that the detection probability of this method can approach to 1 even when the SNR is $-15 \mathrm{~dB}$ while the detection probability of ED is below 0.8 , which means the detection performance of the proposed method can outperform the ED method without the noise variance estimation when the number of antennas is greater than two.
\end{abstract}

\section{Introduction}

A cognitive radio (CR) is a radio which is able to sense the spectral environment over a wide spectrum band and exploit the spectrum usage information to opportunistically provide wireless links that best meet the user communication requirements [1]. Spectrum sensing intends to detect the unused spectrum, and spectrum reallocation intends to share the unused spectrum without introducing harmful interference to other users. In the CR systems, the performance of spectrum sensing will directly affect the spectrum reallocation. Effective spectrum reallocation mainly relies on the good performance of spectrum sensing, and hence, spectrum sensing is considered one of the critical elements in a CR system [2]. The basic function of spectrum sensing in CR systems is to detect the presence of primary users' (PUs) signal. To achieve reliable spectrum sensing, a number of issues have to be taken into account, including low signal-tonoise ratio and multipath fading.

A few classical sensing methods are widely considered for spectrum sensing, including likelihood ratio test (LRT) method [3], matched filtering (MF) method [4], cyclostationary detection (CSD) method [5], and energy detection (ED) method which is one of the most commonly used signal detection methods [6-9]. These classic methods can achieve good sensing performance when given the sufficient information, including PUs' signal waveform, the noise variance, and the cyclic frequency of the PUs' signal. In recent years, many novel spectrum-sensing methods have been proposed to lower the requirement of the above information. For instance, the methods proposed in [10-13] use multiple antennas and the characteristics of covariance matrix to detect the PUs' signal without the prior information about PUs' signal and noise variance. In the CSD 
method, compressive sensing can be used to determine the symmetrical cycle frequency [14], while Fourier transform can be used to obtain the characteristic function of the cycle frequency [15]. In addition, the cooperative spectrum sensing $[16,17]$ can further improve the overall performance of spectrum sensing in CR networks, and some artificial intelligence methods have been also applied in spectrumsensing or spectrum signal classification [18-20].

In this paper, we propose an ED-based blind-sensing method, exploiting the low implementation complexity and without needing any prior information about PUs' signal of the ED method. The proposed method is intended for CR systems with multiple antennas to exploit antenna diversity to get two relevant statistics. One is the original ED decision statistic which is simply defined as the summation of squared amplitude of individual sampled signals over multiple antennas, while the other is defined as the squared norm of the summation of the sampled signal vectors over multiple antennas. The proposed decision statistic is defined as the subtraction of the two statistics divided by a statistic constant so that the constructed decision threshold can be independent of the noise variance. Thus, the decision performance of the proposed method is free of the impact of uncertainty in noise variance, and noise variance estimation is not required which makes this method be a blind-sensing method. Both the ED and the proposed method have a linear computational complexity, although the proposed method has higher computational complexity than ED. However, the proposed method can achieve a significantly better performance than ED without the noise variance estimation in the presence of three or more antennas.

The rest of this paper is organized as follows. The system model is reviewed in Section 2. In Section 3, the classical ED method is reviewed. In Section 4, the details of the proposed method are described. Section 5 gives the simulation results, and Section 6 concludes the paper.

\section{System Model}

In the cognitive radio network, users are divided into two categories: PUs and SUs. The detection of PUs' signal in spectrum sensing can be seen as a binary hypothetical testing problem with hypotheses $H_{0}$ and $H_{1}$ defined as [2]

$$
\left\{\begin{array}{l}
H_{0}: \text { PUs do not exist, } \\
H_{1}: \text { PUs exist. }
\end{array}\right.
$$

When starting to sense the spectrum during the sensing period, a SU will take a certain number $N$ of signal samples to make a decision on whether a PU exists. It is assumed that the $\mathrm{SU}$ receiver has $M$ isotropic antennas, where $M>1$. The signal $r_{i}(n)$ received at the $i$ th antenna can be written as

$$
r_{i}(n)=h_{i} \times s(n)+\eta_{c i}(n),
$$

where $s(n)$ is the PU signal with zero mean which is $E$ $[s(n)]=0$, and in hypothesis $H_{0}, s(n)$ is zero, $h_{i}$ is the channel coefficient, and $\eta_{c i}(n)$ is the additive zero mean white complex Gaussian noise with variance $\sigma_{\eta}^{2}$, which means that the noise components $\left\{\eta_{c i}(n)\right\}$ are independent and identically distributed (IID) and $\eta_{c i}(n) \sim \mathrm{CN}\left(0, \sigma_{\eta}^{2}\right)$. In this paper, it is assumed that the phase shift of $h_{i}$ is known to SU, and we will evaluate the performance of the proposed method for both nonfading channel and IID Rayleigh fading channel. Thus, the output $y_{i}(n)$ of the ith antenna can be modeled as [21]

$$
\begin{aligned}
y_{i}(n) & =\frac{h_{i}^{*}}{\left|h_{i}\right|} r_{i}(n)=\left\{\begin{array}{l}
\eta_{i}(n) ; H_{0}, \\
\left|h_{i}\right| \times s(n)+\eta_{i}(n) ; H_{1},
\end{array}\right. \\
& =\left\{\begin{array}{l}
\eta_{i}(n) ; H_{0}, \\
s_{i}(n)+\eta_{i}(n) ; H_{1},
\end{array}\right.
\end{aligned}
$$

where $\left|h_{i}\right|$ is the norm of the channel coefficient $h_{i}, s_{i}(n)$ is the PU signal sampled by antenna $i$, and $\eta_{i}(n) \sim N\left(0, \sigma_{\eta}^{2}\right)$ [22]. In the nonfading channel, $\left|h_{i}\right|$ can be simply set as 1 , while in a Rayleigh fading channel, $\left|h_{i}\right|$ obeys Rayleigh distribution. Note that how to get an estimate of the channel coefficient is beyond the scope of the paper, but it is useful to deal with this issue and analyse the effect of imperfect channel estimation in the future.

The performance of a spectrum-sensing algorithm can usually be evaluated by two probabilities PD and PFA. PFA is the probability of false alarm that $H_{1}$ is assumed when $H_{0}$ is true, while PD denotes the probability of detection that $H_{1}$ is accepted when $H_{1}$ is true. They are defined as

$$
\begin{aligned}
& P_{f}=P_{r}\left\{\left(y(n): H_{1}\right) \mid H_{0}\right\}, \\
& P_{d}=P_{r}\left\{\left(y(n): H_{1}\right) \mid H_{1}\right\} .
\end{aligned}
$$

Note that PFA is short for the probability of false alarm, and PD is short for the probability of detection, while $P_{d}$ and $P_{f}$ are the variables of PD and PFA. In CR, PFA is a measure of the wasting of spectrum reutilization for $\mathrm{SU}$, while $\mathrm{PD}$ is the measure of noninterference of SU to PU. For a given signal sample size, the detection performance is better if $\mathrm{PD}$ is higher and PFA is lower [12].

\section{Review of the Existing ED Method}

With a given number $N$ of sampled signals $\left\{y_{i}(n)\right\}$, the ED method calculates the total signal power denoted as $T_{\mathrm{ED}}$ and compares it with the threshold $\lambda_{\mathrm{ED}}$ to judge the presence or absence of PU. The ED method performs the hypothesis testing according to

$$
T_{\mathrm{ED}}=\sum_{i=1}^{M} \sum_{n=0}^{N-1}\left|y_{i}(n)\right|^{2} \begin{gathered}
H_{1} \\
< \\
H_{0}
\end{gathered}
$$

where $|x|$ is the norm operation of a complex number $x, N$ is the number of sampled signals $\left\{y_{i}(n)\right\}$ received by the $i$ th antenna, $M$ is the number of antennas, and $\lambda_{\mathrm{ED}}$ is the threshold of ED. The test statistic for ED in a multiple antenna system is equivalent to the output of a square-law combiner (SLC) [8]. $T_{\mathrm{ED}}$ obeys the chi-square distribution 
with $N$ degrees of freedom, and the $\mathrm{PFA}$ of $\operatorname{ED}\left(P_{f, \mathrm{ED}}\right)$ can be written as [7]

$$
P_{f, \mathrm{ED}}=\frac{\Gamma\left(\mathrm{MN} / 2, \lambda_{\mathrm{ED}} / 2 \sigma_{\eta}^{2}\right)}{\Gamma(\mathrm{MN} / 2)}
$$

where $\Gamma(\bullet, \bullet)$ is the incomplete gamma function and $\Gamma(\bullet)$ is the gamma function and $\sigma_{\eta}^{2}$ is the noise variance assumed to be known to SU. Assuming that $N$ (for example, $N>250$ ) [23] is large enough and the channel model is a nonfading channel, the chi-square distribution with $N$ degrees of freedom can be considered as a normal distribution by using the central limit theorem (CLT). Then, $\mathrm{P}_{f, \text { ED }}$ and $\mathrm{P}_{d, \mathrm{ED}}$ can be written as [10]

$$
\begin{aligned}
& P_{f, \mathrm{ED}}=Q\left(\frac{\lambda_{\mathrm{ED}}-\mathrm{MN} \sigma_{\eta}^{2}}{\sigma_{\eta}^{2} \sqrt{2 \mathrm{MN}}}\right), \\
& P_{d, \mathrm{ED}}=Q\left(\frac{\lambda_{\mathrm{ED}}-\mathrm{MN} \sigma_{\eta}^{2} \times(1+\mathrm{SNR})}{\sigma_{\eta}^{2} \sqrt{2 \mathrm{MN}(1+\mathrm{SNR})}}\right),
\end{aligned}
$$

where $Q(\bullet)$ is the $Q$ function and SNR is the signal-to-noise ratio, defined as

$$
\mathrm{SNR}=\frac{P_{s}}{\sigma_{\eta}^{2}}=\frac{\sum_{i=1}^{M} \sum_{n=0}^{N-1}\left|s_{i}(n)\right|^{2}}{\mathrm{MN} \sigma_{\eta}^{2}},
$$

where $P_{s}$ is the average power of the PU signal and $\left\{s_{i}(n)\right\}$ are the PU signal received by antenna $i$. Equation (7) indicates that the threshold $\lambda_{\mathrm{ED}}$ can be obtained by setting PFA $\left(\mathrm{P}_{f, \mathrm{ED}}\right)$ according to the specific requirement when antennas number $M$, sample number $N$, and the noise variance $\sigma_{\eta}^{2}$ are known; meanwhile, equation (8) indicates that the threshold $\lambda_{\mathrm{ED}}$ will directly affect the detection performance of ED which means the selection of the threshold $\lambda_{\mathrm{ED}}$ is the key of ED.

\section{Energy Detection-Based Spectrum-Sensing Method with Multiple Antennas (ED-BS)}

In the proposed method, the decision statistic is constructed as follows. The $N$ sampled signal $\left\{y_{i}(n)\right\}$ can be grouped to form a signal vector as

$$
\mathbf{Y}_{i}=\left[y_{i}(0), y_{i}(1), y_{i}(2), \ldots, y_{i}(N-1)\right],
$$

and then, $T_{\mathrm{ED}}$ can be rewritten from equation (5) as

$$
T_{\mathrm{ED}}=\sum_{i=1}^{M} \sum_{n=0}^{N-1}\left|y_{i}(n)\right|^{2}=\sum_{i=1}^{M} \mathbf{Y}_{i}^{2},
$$

where $\|\mathbf{x}\|$ is the norm operation of a vector $\mathbf{x}$. The sampled signal vectors from the $M$ antennas can be simply combined as

$$
\mathbf{Y}=\mathbf{Y}_{1}+\mathbf{Y}_{2}+\cdots+\mathbf{Y}_{M},
$$

which has the following norm:

$$
\begin{aligned}
\mathbf{Y}^{2} & =\left.\sum_{n=0}^{N-1} \sum_{i=1}^{M} y_{i}(n)\right|^{2} \\
& =\sum_{i=1}^{M} \sum_{n=0}^{N-1}\left|y_{i}(n)\right|^{2}+\sum_{n=0}^{N-1} \sum_{i=1}^{M-1} \sum_{j=i+1}^{M}\left[y_{i}(n) y_{j}^{*}(n)+y_{j}(n) y_{i}^{*}(n)\right] \\
& =T_{\mathrm{ED}}+\sum_{n=0}^{N-1} \sum_{i=1}^{M-1} \sum_{j=i+1}^{M}\left[y_{i}(n) y_{j}^{*}(n)+y_{j}(n) y_{i}^{*}(n)\right] .
\end{aligned}
$$

According to the CLT, $T_{\mathrm{ED}}$ in equation (11) and $\|\mathrm{Y}\|^{2}$ in equation (13) approximately have a normal distribution provided that $N$ is large and the channel gains are random but have the same probability distribution function. The same probability distribution approximation could still be produced even if the antenna gains are quite different. Comparing equation (13) with equation (11), it clearly shows that the norm of simple combination $\mathrm{Y}$ does contain not only the total signal power $T_{\mathrm{ED}}$ but also the crosscorrelation product of the sampled signals by pairs of antennas whose characteristics will be exploited. Subtracting equation (13) from equation (11) and making use of equation (2) produce

$$
\begin{aligned}
\mathbf{Y}^{2}-T_{\mathrm{ED}} & =\sum_{n=0}^{N-1} \sum_{i=1}^{M-1} \sum_{j=i+1}^{M}\left[y_{i}(n) y_{j}^{*}(n)+y_{j}(n) y_{i}^{*}(n)\right], \\
& =\left\{\begin{array}{l}
\sum_{n=0}^{N-1} \sum_{i=1}^{M-1} \sum_{j=i+1}^{M}\left[\eta_{i}(n) \eta_{j}^{*}(n)+\eta_{j}(n) \eta_{i}^{*}(n)\right] ; H_{0}, \\
\left.\sum_{n=0}^{N-1} \sum_{i=1}^{M-1} \sum_{j=i+1}^{M}\left\{\left[\left|h_{i}\right| s_{i}(n)+\eta_{i}(n)\right]\left[\left|h_{j}\right| s_{j}(n)+\eta_{j}(n)\right]^{*}+\left[\left|h_{j}\right| s_{j}(n)+\eta_{j}(n)\right]\left[\left|h_{i}\right| s_{i}(n)+\eta_{i}(n)\right]^{*}\right]\right\} ; H_{1},
\end{array}\right.
\end{aligned}
$$


Equation (14) shows that, in hypothesis $H_{0}$, the difference only contains the noise terms, and in hypothesis $H_{1}$, the difference contains both signal and noise terms. The means of signal $s(n)$ and noise $\eta_{i}(n)$ are zero; thus, in statistics, when $N$ is large enough, the mean of $T_{\mathrm{ED}}$ has [3]

$$
\begin{aligned}
E\left(T_{\mathrm{ED}}\right) & =\frac{\sum_{i=1}^{M} \sum_{n=0}^{N-1}\left|y_{i}(n)-0\right|^{2}}{\mathrm{MN}} \\
& =\frac{\sum_{i=1}^{M} \sum_{n=0}^{N-1}\left|y_{i}(n)-E\left(y_{i}(n)\right)\right|^{2}}{\mathrm{MN}}=\operatorname{var}\left[y_{i}(n)\right],
\end{aligned}
$$

where $\operatorname{var}(x)$ is the operation of taking the variance of $x$.

Now, a new decision variable $T_{\mathrm{ED}-\mathrm{BS}}$ can be defined as

$$
T_{\mathrm{ED}-\mathrm{BS}}=\frac{\mathrm{Y}^{2}-T_{\mathrm{ED}}}{\mathrm{MN} \times \operatorname{var}\left[y_{i}(n)\right]} .
$$

The new decision variable $T_{\mathrm{ED}-\mathrm{BS}}$ can be considered as a new statistic, and then, the decision rule which is similar to the ED method to distinguish the two hypotheses $H_{0}$ and $H_{1}$ from each other is given by

$$
T_{\mathrm{ED}-\mathrm{BS}}=\frac{\mathbf{Y}^{2}-T_{\mathrm{ED}}}{\mathrm{MN} \times \operatorname{var}\left[y_{i}(n)\right]} \stackrel{\stackrel{H_{1}}{>}}{<} \lambda_{\mathrm{ED}-\mathrm{BS}} .
$$

Considering the hypothesis $H_{0}, y_{i}(n)$ is just the Gaussian noise $y_{i}(n)=\eta_{i}(n) \sim N\left(0, \sigma_{\eta}^{2}\right)$; thus, according to CLT, we can readily get that $\|Y\|^{2}-T_{\mathrm{ED}} \sim N\left(0,2 N\left(M^{2}-M\right) \sigma_{\eta}^{2}\right)$ when $N$ is large enough, and the variance of $y_{i}(n)$ is given by

$$
\operatorname{var}\left[y_{i}(n)\right]=E\left(T_{\mathrm{ED}} \mid H_{0}\right)=\frac{\sum_{i=1}^{M} \sum_{n=0}^{N-1}\left|\eta_{i}(n)\right|^{2}}{\mathrm{MN}}=\sigma_{\eta}^{2},
$$

and then, the expectation and variance of $T_{\mathrm{ED}-\mathrm{BS}}$ are

$$
\begin{aligned}
E\left(T_{\mathrm{ED}-\mathrm{BS}}\right) & =E\left(\frac{\mathrm{Y}^{2}-T_{\mathrm{ED}}}{\mathrm{MN} \times \operatorname{var}\left[y_{i}(n)\right]}\right)=0, \\
\operatorname{var}\left[T_{\mathrm{ED}-\mathrm{BS}}\right] & =\operatorname{var}\left(\frac{\mathrm{Y}^{2}-T_{\mathrm{ED}}}{\mathrm{MN} \times \operatorname{var}\left[y_{i}(n)\right]}\right) \\
& =\frac{2 M(M-1) N \sigma_{\eta}^{4}}{M^{2} N^{2} \sigma_{\eta}^{4}}=\frac{2(M-1)}{\mathrm{MN}} .
\end{aligned}
$$

According to CLT, we know that $T_{\mathrm{ED}-\mathrm{BS}}$ can be considered to obey a normal distribution with the expectation 0 and variance $2(M-1) /(\mathrm{MN})$; when $N$ is large enough, $T_{\mathrm{ED}-\mathrm{BS}} \sim N(0,2(M-1) /(\mathrm{MN}))$. Then, PFA of this method $P_{f}$, ED-BS is

$P_{f, \mathrm{ED}-\mathrm{BS}}=P_{r}\left\{T_{\mathrm{ED}-\mathrm{BS}}>\lambda_{\mathrm{ED}-\mathrm{BS}} \mid H_{0}\right\}=\mathrm{Q}\left(\frac{\lambda_{\mathrm{ED}-\mathrm{BS}}-0}{\sqrt{2(M-1) / \mathrm{MN}}}\right)$.

$$
\lambda_{\mathrm{ED}-\mathrm{BS}}=\sqrt{\frac{2(M-1)}{\mathrm{MN}}} \mathrm{Q}^{-1}\left(P_{f, \mathrm{ED}-\mathrm{BS}}\right) .
$$

Equation (22) indicates that the threshold $\lambda_{\mathrm{ED}-\mathrm{BS}}$ is independent of the noise variance so that when the values of $N, M$, and $P_{f \text {, ED-BS }}$ keep constant and $\lambda_{\text {ED-BS }}$ keeps constant too, no matter how big the noise variance is.

Now, considering ED, the threshold $\lambda_{\mathrm{ED}}$ can be obtained by equation (7):

$$
\lambda_{\mathrm{ED}}=\sigma_{\eta}^{2}\left[\mathrm{MN}+\sqrt{2 \mathrm{MN}} \mathrm{Q}^{-1}\left(P_{f, \mathrm{ED}}\right)\right] .
$$

Equation (23) indicates that $\mathrm{PFA}\left(P_{f, \mathrm{ED}}\right), M$, and $N$ are required to determine the threshold for $\mathrm{SU}$ like the proposed method; however, unlike the proposed method, the noise variance is also required. In fact, PFA, $M$, and $N$ can be set by SU according to the specific requirement, but the noise variance needs to be estimated for ED. Assume that $\sigma_{z}^{2}$ is the noise variance estimate used in the ED method, which is different from the real noise variance $\sigma_{\eta}^{2}$. Then, the noise uncertainty factor [12] $U$ can be defined as

$$
U=\frac{\sigma_{z}^{2}}{\sigma_{\eta}^{2}}
$$

Then, $\lambda^{\prime}{ }_{\mathrm{ED}}$ can be written as

$$
\lambda_{\mathrm{ED}}^{\prime}=\sigma_{z}^{2}\left[\mathrm{MN}+\sqrt{2 \mathrm{MN}} Q^{-1}\left(P_{f, \mathrm{ED}}\right)\right]=U \lambda_{\mathrm{ED}} \text {. }
$$

It is clear that when $U=1$, equation (25) is exactly the same as equation (23). However, when $U$ is not equal to one, for a given PFA $\left(P_{f, \text { ED }}\right)$, the threshold calculated by equation (25) will not be the desired threshold which is calculated by equation (23). That is, for ED, the actual PFA ( $\left.P_{f, \text { ED }}^{\prime}\right)$ after performing hypothesis testing is different from the given PFA $\left(P_{f, \text { ED }}\right)$, while for the proposed method ED-BS, the actual PFA will be the same as the given one due to the nonaffection of noise variance. Thus, the noise uncertainty cannot make any impact on $\lambda_{\mathrm{ED}-\mathrm{BS}}$ and $P_{f, \mathrm{ED}-\mathrm{BS}}$ unlike ED.

In the hypothesis $H_{1}$, first, consider the nonfading case; thus, $\left|h_{i}\right|$ in equation (3) will be constant, and for simplicity, set it at 1 . When $N$ is large enough, the variance of $y_{i}(n)$ is

$$
\begin{aligned}
\operatorname{var}\left[y_{i}(n)\right] & =E\left(T_{\mathrm{ED}} \mid H_{1}\right)=\frac{\left.\sum_{i=1}^{M} \sum_{n=0}^{N-1} \mid s_{i}(n)+\eta_{i}(n)\right)\left.\right|^{2}}{\mathrm{MN}} \\
& =P_{s}+\sigma_{\eta}^{2} .
\end{aligned}
$$

Like $T_{\mathrm{ED}},\|Y\|^{2}$ also obeys the noncentral chi-square distribution with $N$ degrees of freedom. And according to CLT, when $N$ is large enough, $\|Y\|^{2}$ can be considered to approximately obey a normal distribution, which is $\|Y\|^{2} \sim N\left(M^{2} \mathrm{NP}_{\mathrm{s}}+\mathrm{NM} \sigma_{\eta}^{2}, 2 \mathrm{NM}^{2} \sigma_{\eta}^{4}+4 \mathrm{NM}^{2} P_{\mathrm{s}} \sigma_{\eta}^{2}\right)$. Then, we can easily obtain

$T_{\mathrm{ED}-\mathrm{BS}} \sim N\left(\frac{(M-1) \mathrm{SNR}}{1+\mathrm{SNR}}, \frac{2(M-1)+4\left(M^{2}-1\right) \mathrm{SNR}}{\mathrm{MN}(1+\mathrm{SNR})^{2}}\right)$.

Then, the threshold $\lambda_{\mathrm{ED}-\mathrm{BS}}$ is calculated by 
Then, PD of this method $P_{d, \mathrm{ED}-\mathrm{BS} \text {,nonfading }}$ is

$$
\begin{aligned}
P_{d, \mathrm{ED}-\mathrm{BS}, \text { nonfading }} & =P_{r}\left\{T_{\mathrm{ED}-\mathrm{BS}}>\lambda_{\mathrm{ED}-\mathrm{BS}} \mid H_{1}\right\} \\
& =Q\left(\frac{\lambda_{\mathrm{ED}-\mathrm{BS}}-(M-1) \mathrm{SNR} / 1+\mathrm{SNR}}{\sqrt{2(M-1)+4\left(M^{2}-1\right) \mathrm{SNR} / \mathrm{MN}(1+\mathrm{SNR})^{2}}}\right) \\
& =Q\left(\frac{(1+\mathrm{SNR}) \lambda_{\mathrm{ED}-\mathrm{BS}}-(M-1) \mathrm{SNR}}{\sqrt{2(M-1)+4\left(M^{2}-1\right) \mathrm{SNR} / \mathrm{MN}}}\right) .
\end{aligned}
$$

It is clear that, as the number $M$ of antennas increases, the $Q$ function in equation (28) increases, and hence, PD increases too. For example, when the values of $N, S N R$, and $P_{f \text {, ED-BS }}$ are set as $1000,-15 \mathrm{~dB}$, and 0.1 , respectively, and the theoretical value of $P_{d}$, ED-BS, nonfading calculated by using equations (23) and (28) is $0.3839,0.6429$, and 0.8371 , when $M$ is 2,3 , and 4 , respectively.

For ED, when the noise variance estimate may be different from the real noise variance, the threshold used in the hypothesis testing is $\lambda_{\mathrm{ED}}^{\prime}$ contained with the noise uncertainty. Then, substituting equation (25) into equation (9), it has

$$
\begin{aligned}
P_{d, \mathrm{ED}}^{\prime} & =Q\left(\frac{\lambda_{\mathrm{ED}}^{\prime}-\mathrm{MN} \sigma_{\eta}^{2} \times(1+\mathrm{SNR})}{\sigma_{\eta}^{2} \sqrt{2 \mathrm{MN}(1+\mathrm{SNR})}}\right) \\
& =Q\left(\frac{U \lambda_{\mathrm{ED}}-\mathrm{MN} \sigma_{\eta}^{2} \times(1+\mathrm{SNR})}{\sigma_{\eta}^{2} \sqrt{2 \mathrm{MN}(1+\mathrm{SNR})}}\right) .
\end{aligned}
$$

Although both equations (28) and (29) indicate that PD is related to $\mathrm{SNR}$, only the decision variables ( $T_{\mathrm{ED}-\mathrm{BS}}$ and $\left.T_{\mathrm{ED}}\right)$ and the thresholds $\left(\lambda_{\mathrm{ED}-\mathrm{BS}}\right.$ and $\left.\lambda_{\mathrm{ED}}^{\prime}\right)$ are needed to perform the hypothesis testing. Then, equation (29) also indicates that the noise uncertainty can make a great impact on the PD of ED $\left(P_{d, \mathrm{ED}}^{\prime}\right)$. As a result, for a given PFA, PD would not be maximized or the actual PFA is greater than the desired one [21], which would be typically not desirable. With the comparison of equations (28) and (29), it is clear that PD of the proposed method ED-BS has no affection of noise uncertainty; for the noise, variance is not required to determine the threshold $\lambda_{\mathrm{ED}-\mathrm{BS}}$ used in the hypothesis testing. Thus, the noise variance estimation is not needed for this method.

In the IID Rayleigh fading case, $\left|h_{i}\right|$ obeys the Rayleigh distribution. For $T_{\mathrm{ED}-\mathrm{BS}}$, it is a challenge to obtain the distribution function directly, but we can use the expectation expression to estimate the SNR for $T_{\mathrm{ED}-\mathrm{BS}}$ and $\mathrm{PD}$. As given in equation (16), the denominator of $T_{\mathrm{ED}-\mathrm{BS}}$ is already in the form of expectation. And the variance of $y_{i}(n)$ can be written as

$$
\begin{aligned}
\operatorname{var}\left[y_{i}(n)\right] & =E\left(T_{\mathrm{ED}} \mid H_{1}\right)=\frac{\left.\sum_{i=1}^{M} \sum_{n=0}^{N-1}|| h_{i} \mid s_{i}(n)+\eta_{i}(n)\right)\left.\right|^{2}}{M N} \\
& =\frac{\sum_{i=1}^{M}\left[\gamma_{i} \mathrm{SNR}+N \sigma_{\eta}^{2}\right]}{\mathrm{MN}}=\sigma_{\eta}^{2}(1+\gamma \mathrm{SNR}),
\end{aligned}
$$

where $\gamma_{i}$ is the total channel gain of antenna $i, \gamma_{i}$ SNR is the SNR of antenna $i$, and $\gamma$ is the average channel gain of all antennas, which is [7]

$$
\gamma=\frac{\sum_{i=1}^{M} \gamma_{i}}{M}
$$

Both $\gamma$ and $\gamma_{i}$ obey the same probability distribution function which is [23]

$$
f(\gamma)=\frac{1}{2} \exp \left(\frac{-\gamma}{2}\right), \quad \gamma>0
$$

Here, for simplicity, the mean of $\gamma$ is assumed as 1 . Then, the expectation and variance of the numerator part of $T_{\mathrm{ED}-\mathrm{BS}}$ can be estimated as

$$
\begin{aligned}
E\left(\mathbf{Y}^{2}-T_{\mathrm{ED}}\right) & \approx E\left[2 \sum_{n=0}^{N-1} \sum_{i=1}^{M-1} \sum_{j=i+1}^{M}\left|h_{i} h_{j} s(n)\right|^{2}\right] \\
& \approx \sum_{n=0}^{N-1}\left[M(M-1)\left|E\left(\left|h_{i}\right|\right) s(n)\right|^{2}\right] \\
& =\frac{\pi}{4} \sigma_{\eta}^{2} \mathrm{NM}(M-1) \mathrm{SNR}, \\
\operatorname{var}\left(\mathbf{Y}^{2}-T_{\mathrm{ED}}\right) & =E\left\{\left[Y^{2}-T_{\mathrm{ED}}-E\left(Y^{2}-T_{\mathrm{ED}}\right)\right]^{2}\right\} \\
& \approx \sigma_{\eta}^{4} \mathrm{NM}\left[(M-1)+\left(M^{2}-1\right) \pi \mathrm{SNR} .\right.
\end{aligned}
$$

Substituting equations (30), (33), and (34) into equations (27) and (28), we can produce 


$$
\begin{aligned}
T_{\mathrm{ED}-\mathrm{BS}} & \sim N\left(\frac{(M-1) \pi \mathrm{SNR}}{4(1+\gamma \mathrm{SNR})}, \frac{2(M-1)+\left(M^{2}-1\right) \pi \mathrm{SNR}}{\mathrm{MN}(1+\gamma \mathrm{SNR})^{2}}\right), \\
P_{d, \mathrm{ED}-\mathrm{BS}, \text { rayleigh }} & =P_{r}\left\{T_{\mathrm{ED}-\mathrm{BS}}>\lambda_{\mathrm{ED}-\mathrm{BS}} H_{1} \mid\right\} \\
& =\int_{0}^{\infty} \mathrm{Q}\left(\frac{(1+\gamma \mathrm{SNR}) \lambda_{\mathrm{ED}-\mathrm{BS}}-\pi / 4(M-1) \mathrm{SNR}}{\sqrt{2(M-1)+\left(M^{2}-1\right) \pi \mathrm{SNR} / \mathrm{MN}(1+\gamma \mathrm{SNR})^{2}}}\right) f(\gamma) \mathrm{d} \gamma .
\end{aligned}
$$

It would be difficult, if not impossible, to obtain a closed from expression for the integral in equation (36). Thus, the Gauss-Laguerre quadrature method [24] is employed to obtain an approximate solution, which is

$$
\int_{0}^{\infty} e^{-x} f(x) \mathrm{d} x \approx \sum_{i=1}^{n} w_{i} f\left(x_{i}\right)
$$

where $w_{i}$ are the weight coefficients, as listed in Table 1.

Then, substituting equations (32) and (37) into (36) and using the variable change $x=\gamma / 2$, we produce

$$
\begin{aligned}
P_{d, \mathrm{ED}-\mathrm{BS}, \text { rayleigh }} & =\int_{0}^{\infty} \mathrm{Q}\left(\frac{(1+2 x \mathrm{SNR}) \lambda_{\mathrm{ED}-\mathrm{BS}}-\pi / 4(M-1) \mathrm{SNR}}{\sqrt{2(M-1)+\left(M^{2}-1\right) \pi \mathrm{SNR} / \mathrm{MN}(1+\gamma \mathrm{SNR})^{2}}}\right) e^{-x} \mathrm{~d} x \\
& \approx \sum_{i=1}^{5} w_{i} \mathrm{Q}\left(\frac{\left(1+2 x_{i} \mathrm{SNR}\right) \lambda_{\mathrm{ED}-\mathrm{BS}}-\pi / 4(M-1) \mathrm{SNR}}{\sqrt{2(M-1)+\left(M^{2}-1\right) \pi \mathrm{SNR} / \mathrm{MN}(1+\gamma \mathrm{SNR})^{2}}}\right) .
\end{aligned}
$$

Note that equations (35), (36), and (38) are just the approximate estimation formulas, which are only used for estimating the detection performance of this proposed method. Equations (28) and (36) indicate that PD of this method is a function of the threshold $\lambda_{\mathrm{ED}-\mathrm{BS}}$ such as the ED method, but equation (22) shows that the threshold $\lambda_{\mathrm{ED}-\mathrm{BS}}$ is independent of the noise variance, while the threshold $\lambda_{\mathrm{ED}}$ is dependent of the noise variance. Once $M, N$, and $P_{f \text {, ED-BS }}$ and SNR keep constant, the PD and threshold of this proposed method will keep constant and cannot be impacted by the accuracy of noise variance estimation unlike the ED method. Thus, the noise estimation process is not needed for this method.

It is well known that the ED method has a low linear computational complexity, which is usually considered as $\mathrm{O}(N)$. Comparing equation (17) with equation (5), it is clear that this proposed method has $\mathrm{MN}-1$ more addition operations, one more division operation, one more subtraction operation, and $\mathrm{N}$ more squaring operations than ED which only needs $\mathrm{MN}-1$ addition and $\mathrm{MN}$ squaring operations. The total computation complexity of this proposed method is $O(3 \mathrm{MN}+N+2)$, while $\mathrm{ED}$ is $\mathrm{O}(2 \mathrm{MN}-1)$. It means that the computational complexity of this method is almost twice that of ED shown in Table 2. If $M$ is not very big $(M<<N)$, the computational complexity of this method can be approximated as $\mathrm{O}(N)$, indicating that this proposed method has a linear computational complexity such as ED.

\section{Simulation Results}

Here, the simulation results are given to evaluate the performance of the proposed method. It is assumed that the PU signal is the BPSK signal with central frequency $f_{c}=20 \mathrm{MHz}$ and the data transfer rate $R=20 \mathrm{kbps}$, and the sampling frequency is 200 kilo samples per second and the Gaussian noise variance is $\sigma_{\eta}^{2}=1$. There are 1000 trails for each experiment. We will compare the performance of the proposed method (ED-BS) with that of the ED method. The theoretical performance simulation of ED and this proposed method can be obtained by equations (8) and (28).

Figure 1- 3 show PD versus SNR for ED and the proposed method (ED-BS), when PFA is set as 0.1 and $N$ is 1000. In the nonfading case, Figure 1 shows that the simulated PD of this method is very close to its theoretical results which are obtained by equation (28). In Figure 2, when SNR is $-15 \mathrm{~dB}$, the PD of the proposed method is $0.38,0.637$, and 0.84 for two, three, and four antennas, respectively, while the PD of ED is $0.34,0.476$, and 0.55 for two, three, and four antennas, respectively. That is, compared to $\mathrm{ED}, \mathrm{PD}$ of this method is increased by $0.04,0.161$, and 0.29 for two, three, and four antennas, respectively. Figure 3 shows the results for the IID Rayleigh fading channel. PD of both ED and this method decreases for the effect of fading, comparing to the nonfading cases. It is clear from Figures 2 and 3 that, with three antennas or more, the PD of this proposed method is still better than $180 \mathrm{ED}$, but with two antennas, the PD of this 
TABle 1: The value of $w_{i}$ and $x_{i}$.

\begin{tabular}{lcc}
\hline & $w_{i}$ & $x_{i}$ \\
\hline$i=1$ & 0.5217556105 & 0.2635603197 \\
$i=2$ & 0.3986668110 & 1.4134030591 \\
$i=3$ & 0.0759424496 & 3.5964257710 \\
$i=4$ & 0.0036117586 & 7.0858100058 \\
$i=5$ & 0.0000233699 & 12.6408008442 \\
\hline
\end{tabular}

TABLE 2: The total computation complexity of ED and ED-BS with different $M$ and $N$.

\begin{tabular}{lcc}
\hline & $\begin{array}{c}\text { The computation } \\
\text { complexity of ED }\end{array}$ & $\begin{array}{c}\text { The computation } \\
\text { complexity of ED-BS }\end{array}$ \\
\hline$M=2, N=500$ & $O(3502)$ & $O(1999)$ \\
$M=3, N=500$ & $O(5002)$ & $O(2999)$ \\
$M=4, N=500$ & $O(6502)$ & $O(3999)$ \\
$M=2, N=1000$ & $O(7002)$ & $O(3999)$ \\
$M=3, N=1000$ & $O(10002)$ & $O(5999)$ \\
$M=4, N=1000$ & $O(13002)$ & $O(7999)$ \\
$M=2, N=2000$ & $O(14002)$ & $O(7999)$ \\
$M=3, N=2000$ & $O(20002)$ & $O(11999)$ \\
$M=4, N=2000$ & $O(26002)$ & $O(15999)$ \\
\hline
\end{tabular}

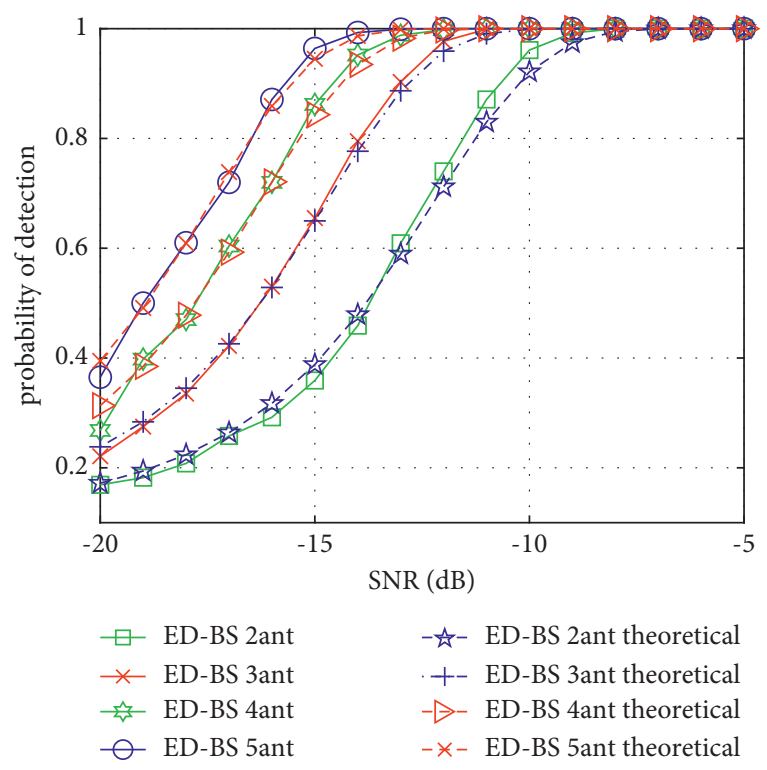

FIGURE 1: Simulated and theoretical detection performance of EDBS with no fading.

method has decreased similarly as ED with one antenna in the Rayleigh fading case. Because the threshold of ED is related to noise variance, the noise variance uncertainty factor $0.5 \mathrm{~dB}(\mathrm{ED}-0.5 \mathrm{~dB})$ is shown in Figures 2 and 3. It has been shown that, without noise uncertainty, PD of ED is better, and with noise uncertainty, PD of ED is almost zero when SNR is below $-10 \mathrm{~dB}$ regardless of whether the channel model is a nonfading channel or Rayleigh fading channel.

Figures 4 and 5 show PD versus $M$ for $\mathrm{ED}$ and the proposed ED-BS, when PFA is set as 0.1 and $N$ is 1000 . It is clear from these figures that, with four or more antennas, the performance of ED-BS is better than that of ED in both the nonfading channel and the Rayleigh fading channel. In the

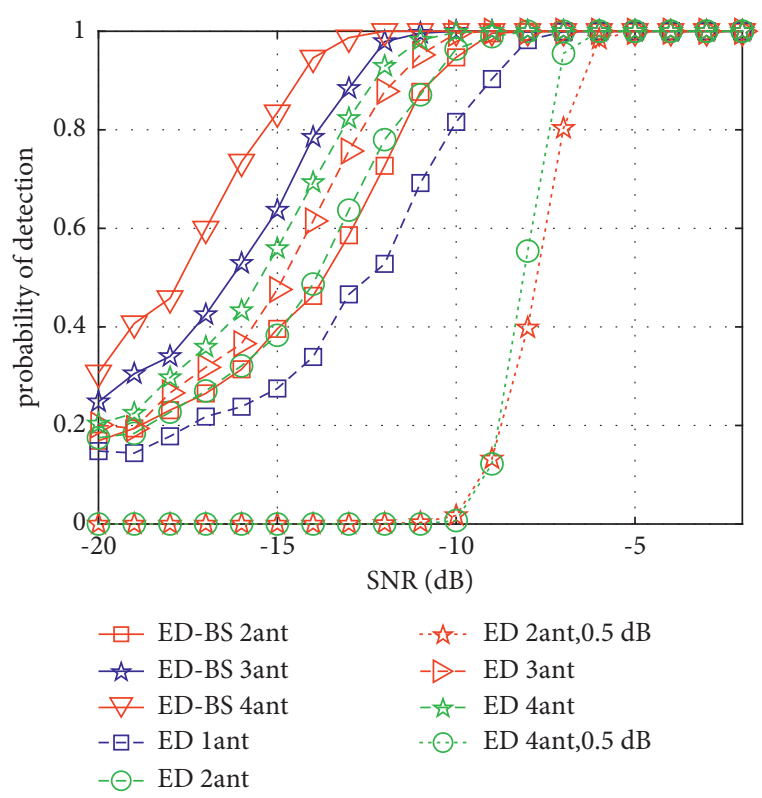

FIgURE 2: Simulated detection performance of ED and ED-BS with no fading.

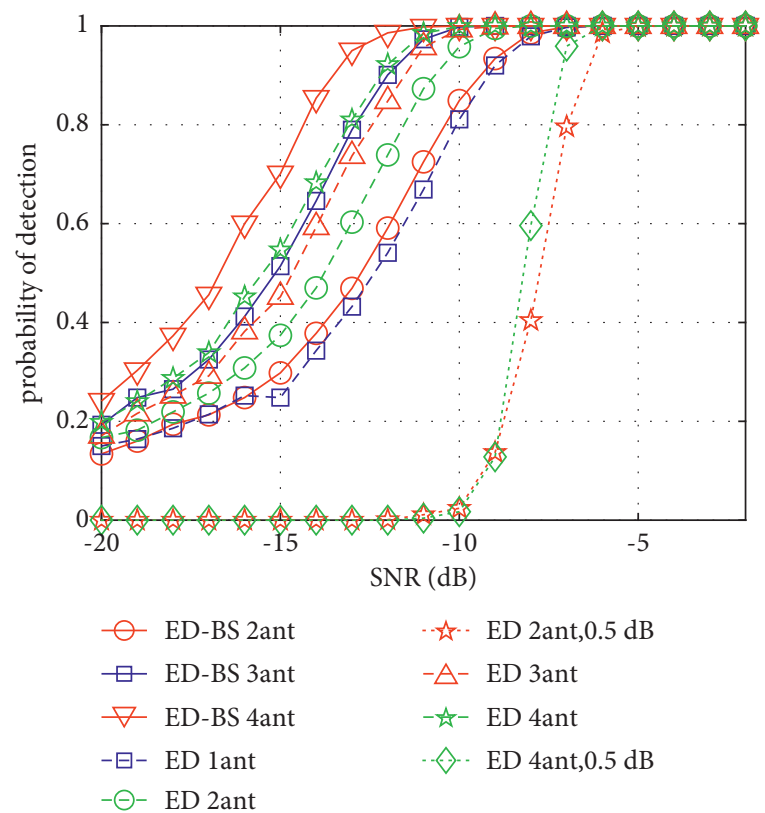

FIGURE 3: Simulated detection performance of ED and ED-BS with Rayleigh fading.

case of three antennas and a Rayleigh fading channel, ED-BS still slightly outperforms ED, while ED has better performance with two antennas in a Rayleigh fading channel. The results also indicate that the greater the number $M$ of antennas is, the higher PD proposed method can achieve.

Figure 6 shows PD versus SNR for ED, ED-BS, and two other blind-sensing methods, blindly combined energy detection (BCED) [12] and eigenvalue Decomposition (EVD) method [10] with four antennas, when PFA is set as 0.1 and $N$ is 1000 . It can be seen from Figure 6 that the PD of the proposed method is much higher than those of the ED 


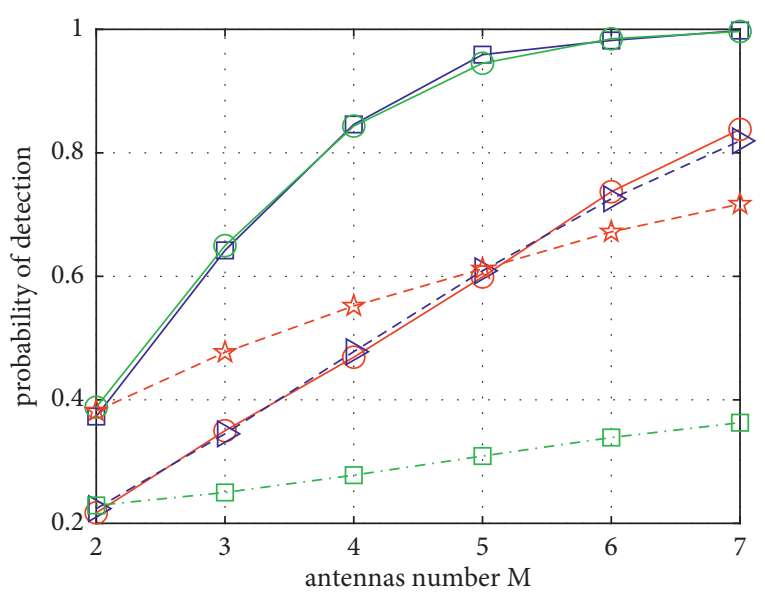

$$
\begin{aligned}
& \square \text { ED-BS }-15 \mathrm{~dB} \quad-\rightarrow \text { - ED-BS }-18 \mathrm{~dB} \text { theoretical } \\
& \bigcirc \text { ED-BS }-18 \mathrm{~dB}-\text { 支- ED }-15 \mathrm{~dB} \\
& \bigcirc \text { ED-BS }-15 \mathrm{~dB} \quad \exists-\mathrm{ED}-18 \mathrm{~dB}
\end{aligned}
$$

FIGURE 4: Simulated detection performance of ED and ED-BS affected by $M$ with no fading.

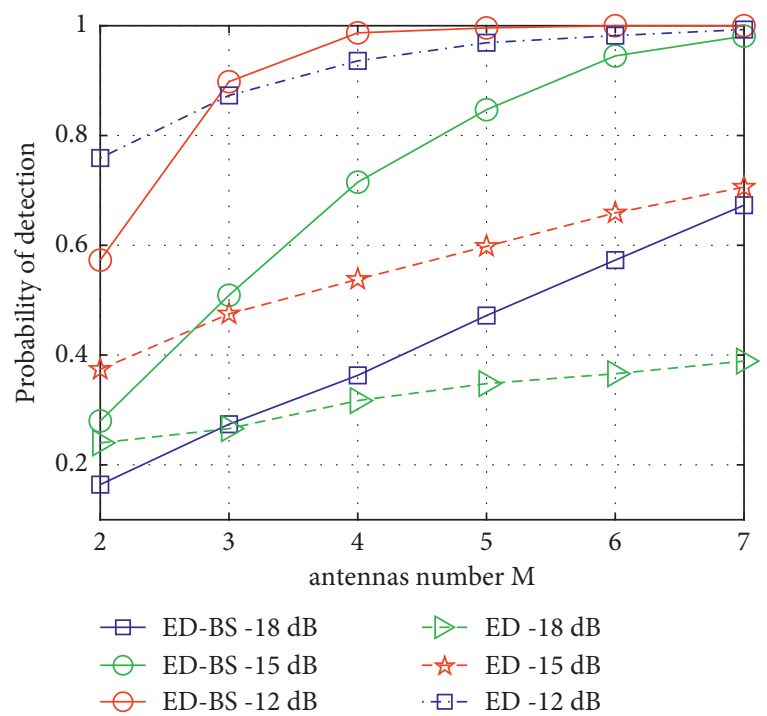

FIGURE 5: Simulated detection performance of ED and ED-BS affected by $M$ with Rayleigh fading.

and the other two blind-sensing methods. In Figure 6, the noise variance is not required to know in advance for the proposed method and two other blind-sensing methods, but the ED method requires knowing it in advance.

Figures 7 and 8 show $P_{d}$ versus $P_{f}$ and this proposed method when the average SNR is $-15 \mathrm{~dB}$ and $N$ is 1000 . Figure 7 is for nonfading channel, while Figure 8 is for IID Rayleigh fading channel. The detection performance of this proposed method with two antennas is close to that of $\mathrm{ED}$ with two antennas in the nonfading case when PFA is not below 0.001, but degrades close to the performance of ED with one antenna in Rayleigh fading case, while the detection performance of this method with four antennas is still much better than that of ED with four antennas.

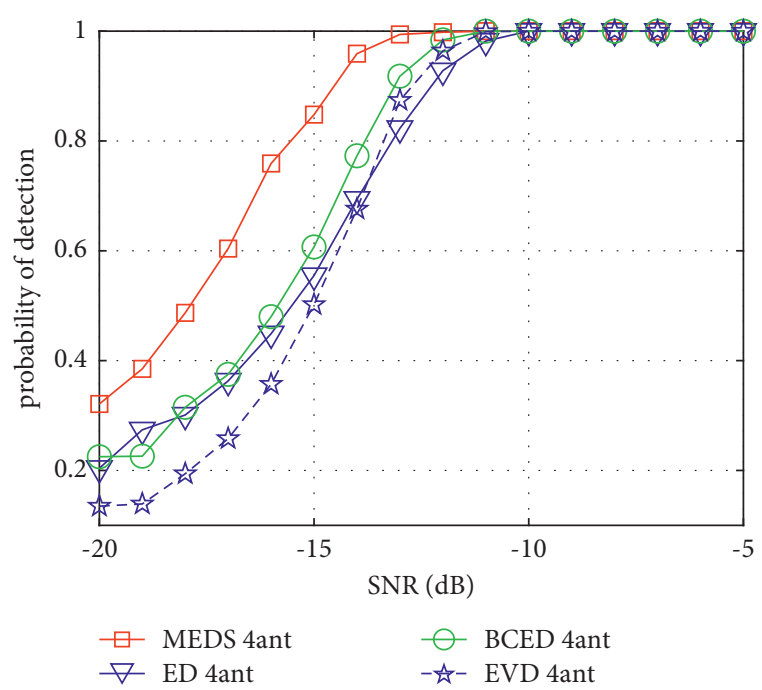

FIGURE 6: Simulated detection performance of ED, BCED, EVD, and ED-BS with no fading.

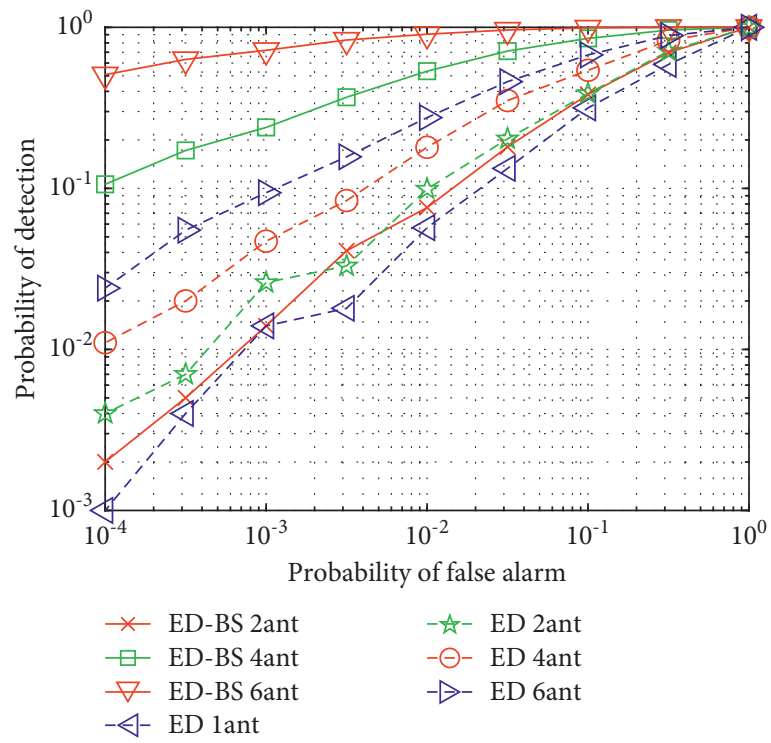

Figure 7: $P_{d}$ versus $P_{f}$ of ED and ED-BS with no fading.

Figure 9 shows the effect of the number $N$ of samples on the detection performance of ED and ED-BS; when $N$ ranges from 500 to 2000 , the average SNR is $-15 \mathrm{~dB}$ and PFA is set as 0.1 , and it is for the nonfading channel case. In the nonfading channel with two antennas, the PD of this proposed method is close to that of ED, as shown in Figures 2, 4, and 7. The simulated performance of this proposed method is very close to its theoretical simulation results in the nonfading channel. With a large $N$, the detection probability of this method approaches to 1 even when the SNR is $-15 \mathrm{~dB}$. This means that the performance of this method can be improved by increasing the number of samples, the number of antennas, or both. Note that there is always a certain deviation between statistical simulation and theoretical values; especially, when the number of antennas is 2 , the approximation of the decision variable $T_{\mathrm{ED}-\mathrm{BS}}$ to 


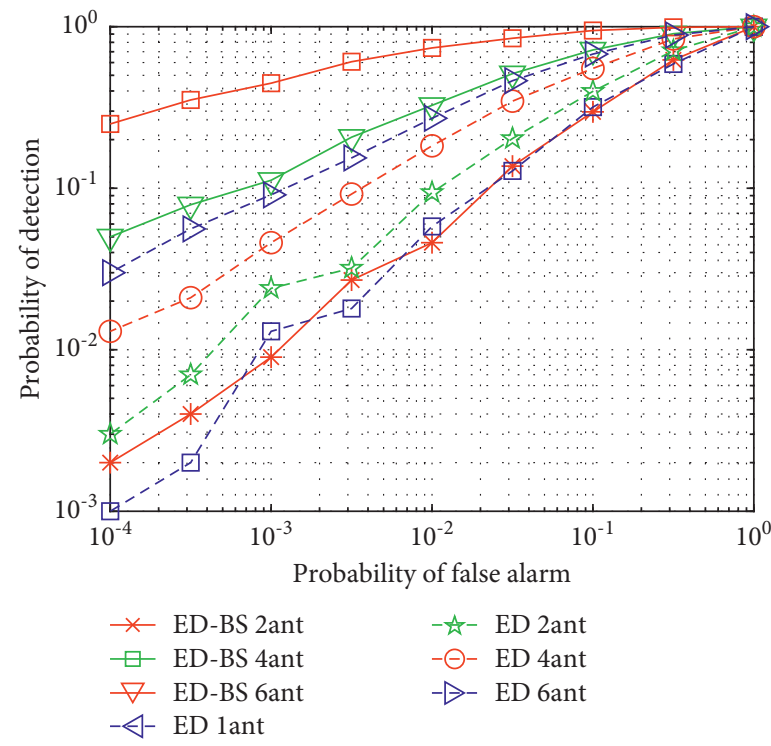

FIgURE 8: $P_{d}$ versus $P_{f}$ of ED and ED-BS with Rayleigh fading.

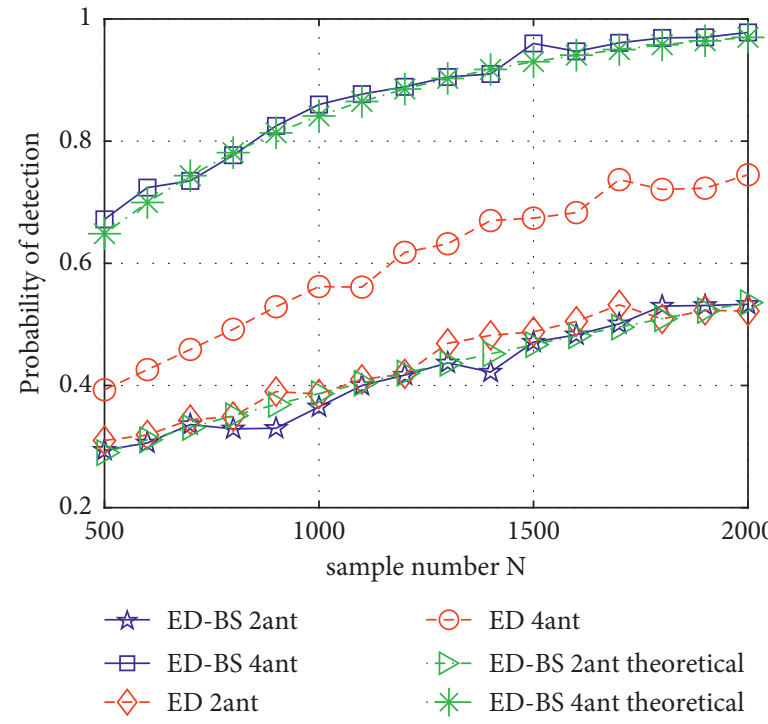

FIgURE 9: Simulated and theoretical detection performance of ED and ED-BS affected by $N$.

TABLE 3: Simulation values and theoretical values of the detection performance of ED-BS with different simulation trails.

\begin{tabular}{lcccccc}
\hline & $\begin{array}{c}1^{\text {st }} \text { simulation } \\
\text { value }\end{array}$ & $\begin{array}{c}2^{\text {nd }} \text { simulation } \\
\text { value }\end{array}$ & $\begin{array}{c}3^{\text {rd }} \text { simulation } \\
\text { value }\end{array}$ & $\begin{array}{c}4^{\text {th }} \text { simulation } \\
\text { value }\end{array}$ & $\begin{array}{c}5^{\text {th }} \text { simulation } \\
\text { value }\end{array}$ & $\begin{array}{c}\text { theoretical } \\
\text { value }\end{array}$ \\
\hline$M=2,1000$ trails & 0.370 & 0.387 & 0.396 & 0.359 & 0.407 & 0.387 \\
$M=4,1000$ trails & 0.828 & 0.871 & 0.865 & 0.846 & 0.870 & 0.841 \\
$M=2,2000$ trails & 0.391 & 0.393 & 0.370 & 0.394 & 0.385 & 0.387 \\
$M=4,2000$ trails & 0.853 & 0.858 & 0.859 & 0.862 & 0.850 & 0.841 \\
$M=2,5000$ trails & 0.371 & 0.382 & 0.380 & 0.376 & 0.384 & 0.387 \\
$M=4,5000$ trails & 0.843 & 0.850 & 0.856 & 0.851 & 0.850 & 0.841 \\
\hline
\end{tabular}

Gaussian distribution will be weaker than that of other antennas. Therefore, when the parameters change, some curves in Figures 7, 8, and 9 may show irregularity in a certain extent, but the overall trend is still consistent with the theoretical prediction. Table 3 lists the simulation values and theoretical values of the detection performance of ED-BS with different simulation trails for 5 times; when $N$ is 1000 , SNR is $-15 \mathrm{~dB}$ and PFA is set as 0.1 , and it is for the nonfading channel case. And it indicates that if the number of simulation trails increases, both the irregularity and the deviation between simulation and theory will decrease. 


\section{Conclusions}

In this paper, a new spectrum-sensing method has been introduced. The proposed method is based on ED, but does not need to estimate the noise variance, since the calculation of the threshold used in this method does not require the knowledge of the noise variance unlike ED. Thus, it can significantly reduce the impact of the noise variance uncertainty. Two classical channel models (Gaussian and Rayleigh fading) are considered. The simulation results have shown that the proposed method significantly outperforms the ED method without the noise variance estimation when there are two or more antennas for a Gaussian channel and three or more antennas for Rayleigh fading channel.

\section{Data Availability}

All data are included within the article.

\section{Conflicts of Interest}

The authors declare that there are no conflicts of interest regarding the publication of this paper.

\section{Acknowledgments}

This work was financially supported by the Project for Science and Technology of Jiangxi Education Department (no. GJJ191048).

\section{References}

[1] J. Mitola, Cognitive radio: an integrated agent architecture for software defined radio, Ph.D. thesis, The Royal Institute of Technology, Stockholm, SE, USA, 2000.

[2] Y.-C. Liang, K.-C. Chen, G. Y. Li, and P. Mahonen, "Cognitive radio networking and communications: an overview," IEEE Transactions on Vehicular Technology, vol. 60, no. 7, pp. 3386-3407, 2011.

[3] S. M. Kay, Fundamentals of Statistical Signal Processing, PTR Prentice Hall, Hoboken, NJ, USA, 1993.

[4] A. Sahai and D. Cabric, "Spectrum sensing fundamental limits and practical challenges," in Proceedings of the IEEE International Symposium on New Frontiers in Dynamic Spectrum Access Networks Proceedings(DySPAN), Baltimore, USA, November 2005.

[5] W. A. Gardner, "Exploitation of spectral redundancy in cyclostationary signals," IEEE Signal Processing Magazine, vol. 8, no. 2, pp. 14-36, 1991.

[6] H. Urkowitz, "Energy detection of unknown deterministic signals," Proceedings of the IEEE, vol. 55, no. 4, pp. 523-531, 1967.

[7] Y. Ye, Y. Li, G. Lu, and F. Zhou, "Improved energy detection with laplacian noise in cognitive radio," IEEE Systems Journal, vol. 13, no. 1, pp. 18-29, 2019.

[8] H. Ju, E. Cho, and S.-H. Kim, "Energy-detection based false alarm reduction in polar-coded uplink control channel transmission in 5G-NR," in Proceedings of the 2021 IEEE 93rd Vehicular Technology Conference (VTC2021-Spring), pp. 1-6, Helsinki, Finland, April 2021.
[9] S. Kumar, "Performance of ED based spectrum sensing over $\alpha-\eta-\mu$ fading channel," Wireless Personal Communications, vol. 100, no. 4, pp. 1845-1857, 2018.

[10] A. Al-Abbasi and T. Fujii, "A novel blind diversity detection scheme for multi-antenna cognitive radio spectrum sensing," in Proceedings of the IEEE 72nd Vehicular Technology Conference Proceedings, pp. 1-5, Ottawa, ON, Canada, September 2010.

[11] P. De and Y. C. Ying-Chang Liang, "Blind spectrum sensing algorithms for cognitive radio networks," IEEE Transactions on Vehicular Technology, vol. 57, no. 5, pp. 2834-2842, 2008.

[12] Y. Yonghong Zeng, Y. C. Rui Zhang, and R. Zhang, "Blindly combined energy detection for spectrum sensing in cognitive radio," IEEE Signal Processing Letters, vol. 15, pp. 649-652, 2008.

[13] L. Shen, H. Wang, W. Zhang, and Z. Zhao, "Multiple antennas assisted blind spectrum sensing in cognitive radio channels," IEEE Communications Letters, vol. 16, no. 1, pp. 92-94, 2012.

[14] L. Safatly, B. Aziz, A. Nafkha et al., "Blind spectrum sensing using symmetry property of cyclic autocorrelation function: from theory to practice," EURASIP Journal on Wireless Communications and Networking, vol. 2014, no. 1, 13 pages, 2014.

[15] W. M. Jang, "Blind cyclostationary spectrum sensing in cognitive radios," IEEE Communications Letters, vol. 18, no. 3, pp. 393-396, 2014.

[16] A. Mohammadi and M. R. Taban, "Cooperative spectrum sensing using fuzzy membership function of energy statistics," AEU-International Journal of Electronics and Communications, vol. 70, no. 3, pp. 234-240, 2016.

[17] O. Simpson and Y. Sun, "Robust statistics evidence based secure cooperative spectrum sensing for cognitive radio networks," in Proceedings of the 2020 International Wireless Communications and Mobile Computing (IWCMC), pp. 1733-1739, Limassol, Cyprus, June 2020.

[18] M. A. Hossain, R. M. Noor, K. A. Yau et al., "Machine learning-based cooperative spectrum sensing in dynamic segmentation enabled cognitive radio vehicular network," Energies, vol. 14, no. 4, pp. 1-30, 2021.

[19] M. Liu, G. Liao, N. Zhao, H. Song, and F. Gong, "Data-driven deep learning for signal classification in industrial cognitive radio networks," IEEE Transactions on Industrial Informatics, vol. 17, no. 5, pp. 3412-3421, 2021.

[20] M. Liu, K. Yang, N. Zhao, Y. Chen, H. Song, and F. Gong, "Intelligent signal classification in industrial distributed wireless sensor networks based industrial internet of things," IEEE Transactions on Industrial Informatics, vol. 17, no. 7, pp. 4946-4956, 2021.

[21] H. Montes, P. Alarcon, R. Ponticelli, and M. Armada, "Performance analysis of primary user detection in a multiple antenna cognitive radio," in Proceedings of the 2007 IEEE International Conference on Communications, pp. 6482-6486, Glasgow, Scotland, June 2007.

[22] R. G. Gallager, Principles of Digital Communication, Cambridge University Press, Cambridge, UK, 2008.

[23] D. M. Martinez and A. G. Andrade, "Adaptive energy detector for spectrum sensing in cognitive radio networks," Computers and Electrical Engineering, vol. 52, pp. 226-239, 2015.

[24] D. Zwillinger, CRC Standard Mathematical Tables and Formulae, CRC Press, Boca Raton, FL, USA, 32nd edition, 2002. 